Virginia Commonwealth University vCU Scholars Compass

2008

\title{
First-principles study of interaction of molecular hydrogen with Li-doped carbon nanotube peapod structures
}

\section{Li Chen}

Rensselaer Polytechnic Institute

Yiming Zhang

Rensselaer Polytechnic Institute

Nikhil Koratkar

Rensselaer Polytechnic Institute

Puru Jena

Virginia Commonwealth University, pjena@vcu.edu

Saroj K. Nayak

Virginia Commonwealth University

Follow this and additional works at: http://scholarscompass.vcu.edu/phys_pubs

Part of the Physics Commons

Chen, L., Zhang, Y., Koratkar, N., et al. First-principles study of interaction of molecular hydrogen with Li-doped carbon nanotube peapod structures. Physical Review B, 77, 033405 (2008). Copyright (C) 2008 American Physical Society.

\section{Downloaded from}

http://scholarscompass.vcu.edu/phys_pubs/68

This Article is brought to you for free and open access by the Dept. of Physics at VCU Scholars Compass. It has been accepted for inclusion in Physics Publications by an authorized administrator of VCU Scholars Compass. For more information, please contact libcompass@vcu.edu. 


\title{
First-principles study of interaction of molecular hydrogen with Li-doped carbon nanotube peapod structures
}

\author{
Li Chen, ${ }^{1}$ Yiming Zhang, ${ }^{1}$ Nikhil Koratkar, ${ }^{2}$ Puru Jena, ${ }^{3}$ and Saroj K. Nayak ${ }^{1}$ \\ ${ }^{1}$ Department of Physics, Applied Physics and Astronomy, Rensselaer Polytechnic Institute, Troy, New York 12180, USA \\ ${ }^{2}$ Mechanical Engineering Department, Rensselaer Polytechnic Institute, Troy, New York 12180, USA \\ ${ }^{3}$ Physics Department, Virginia Commonwealth University, Richmond, Virginia 23284, USA \\ (Received 21 May 2007; revised manuscript received 12 September 2007; published 8 January 2008)
}

\begin{abstract}
Using first-principles density functional theory based on gradient corrected approach, we have studied interaction of $\mathrm{H}_{2}$ molecule with $\mathrm{Li}$-doped carbon nanotube and nanotube based peapod structures. We find that $\mathrm{H}_{2}$ physisorbs on pure carbon nanotube, which is in agreement with earlier studies, and this binding increases when $\mathrm{H}_{2}$ binds to Li-decorated on carbon nanotube surfaces: the binding is further enhanced with $\mathrm{Li}$ atoms deposited on $\mathrm{C}_{60}$ doped nanotube peapod structures. The increase in binding in the latter structures arises due to charge transfer between the nanotube and $\mathrm{C}_{60}$, which further facilitates charge transfer from $\mathrm{Li}$ to the nanotube. Encapsulating fullerene molecule inside the nanotube provides a different way of increasing charge concentration on $\mathrm{Li}$ atom adsorbed outside the nanotube. The increase in $\mathrm{H}_{2}$ binding energy due to $\mathrm{C}_{60}$ encapsulation, compared to recently engineered metal doped nanotube structures, may lead to different carbon based materials for hydrogen storage at room temperature.
\end{abstract}

DOI: 10.1103/PhysRevB.77.033405

PACS number(s): 73.22.-f, 36.40.- c

Carbon based materials show promise for effective hydrogen storage..$^{1}$ In particular, nanoscale carbon structures such as carbon nanotube, fullerenes, and carbon nanofibers, due to their large surface area, have attracted much attention as molecular hydrogen storage materials for meeting U.S. Department of Energy (DOE) target of $9 \mathrm{wt} \%$ and $81 \mathrm{~g} / 1$ (Refs. 2-10) for the year 2015. While early experimental studies showed large $\mathrm{H}_{2}$ wt $\%$ for single walled carbon nanotube, subsequent experiments find that the $\mathrm{H}_{2}$ wt $\%$ for pure carbon nanotube structures may not exceed $1 \mathrm{wt} \% .{ }^{1-13}$ One of the primary reasons for these conflicting results is that the binding of $\mathrm{H}_{2}$ on carbon nanotube surface is van der Waals in nature (binding energy range from 20 to $100 \mathrm{meV}$ ) and the wt $\%$ could be easily changed with slight change in experimental conditions such as temperature and pressure in experiments and/or presence of other molecules such as $\mathrm{H}_{2} \mathrm{O} / \mathrm{OH}^{14-16}$ Recently, attempts have been made to increase the binding of $\mathrm{H}_{2}$ with functionalized carbon nanotube. Yildirim and Ciraci, for example, considered Ti decorated carbon nanotube surface and found that binding of $\mathrm{H}_{2}$ improves considerably to $7 \mathrm{wt} \%$ of hydrogen storage capacity. ${ }^{17}$ Similar conclusions are found when Ti is decorated on fullerene structures. While this approach opens an avenue for storing hydrogen with a good wt $\%$, the work was based on the assumption that Ti prefers to coat the nanotube surface as monolayers without clustering. Sun et al., however, showed in a recent paper that Ti indeed prefers to cluster on these surfaces thereby not being effective in increasing the binding of $\mathrm{H}_{2}$ to the Ti-doped nanotube: the clustering, in addition, reduces the wt $\%$ for hydrogen storage in these doped systems. ${ }^{18}$ They also showed that when Li atoms are decorated on fullerenes, they rather prefer to stay as atoms on these surfaces and clustering of $\mathrm{Li}$ atoms is energetically highly unfavorable. ${ }^{19}$ Subsequently, Sun et al. showed that hydrogen molecule binds to the $\mathrm{Li}$ on nanotube surface with binding energy of $0.075 \mathrm{eV} / \mathrm{H}_{2}$ with $13 \mathrm{wt} \%{ }^{19}$ These results suggest that Li-doped carbon nanostructures may not only improve molecular hydrogen interaction with the mate- rial but also give higher wt \%. Subsequently, Cabria et al. have studied interaction of $\mathrm{H}_{2}$ with Li-doped graphene and carbon nanotube using first-principles density functional method. ${ }^{20}$ The authors in both the above papers ${ }^{19,20}$ find that while the binding energy of $\mathrm{H}_{2}$ with $\mathrm{Li}$ doped system increases compared to pure carbon systems, the interaction energy $(\sim 100 \mathrm{meV})$ is below what would be required for room temperature hydrogen adsorption and desorption process $(\sim 300 \mathrm{meV})$ as needed by DOE.

In this Brief Report, we have studied interaction of $\mathrm{H}_{2}$ with Li-doped carbon nanotube peapod structures using firstprinciples density functional method. We find that binding of $\mathrm{H}_{2}$ with $\mathrm{Li}$ deposited on peapod complex is increased substantially compared to that on Li-doped carbon nanotube and is about eight times that on pure nanotube structure. The increase in binding arises due to charge transfer from nanotube to encapsulated fullerenes, which, in turn, increases charge transfer from $\mathrm{Li}$ to nanotube surface. This makes $\mathrm{Li}$ more positively charged, resulting in stronger charge induced dipole interaction between $\mathrm{H}_{2}$ and $\mathrm{Li}$. The large increase in $\mathrm{H}_{2}$ binding with carbon nanotube based peapod structures compared to that with the previous carbon nanotube based structures suggests a different class of systems for future hydrogen storage materials. The remaining of this Brief Report is organized as follows. In the next section, we present the computational details. In Sec. III, we present our results followed by the conclusions in Sec. IV.

Our calculations are based on first-principles density functional method. In particular, we have used generalized gradient approximation (GGA) of Perdew and Wang $(\mathrm{PW} 91)^{21}$ for exchange and correlation functional. The calculations are based on supercell approach and the wave functions are expanded using plane waves. The ionic cores are replaced by ultrasoft pseudopotentials ${ }^{22}$ and the accuracy of pseudopotentials scheme is tested for carbon system by comparing the results with that obtained using projector augmented wave method. ${ }^{23}$ We have used an energy cutoff of $330 \mathrm{eV}$ and the Monkhorst-pack scheme for $k$-point sam- 
TABLE I. Comparison of accuracy of DFT method with MP2 results for $\mathrm{H}_{2}$ interaction with graphite surface.

\begin{tabular}{lcc}
\hline \hline Method & $\begin{array}{c}\text { Graphite } \mathrm{H}_{2} \\
\text { distance }(\AA)\end{array}$ & $\begin{array}{c}\text { Binding energy } \\
(\mathrm{meV})\end{array}$ \\
\hline MP2 $^{\mathrm{a}}$ & $\sim 3$ & 90 \\
Present calculation based on DFT & 3.07 & 23 \\
\hline \hline
\end{tabular}

${ }^{a}$ Reference 26.

pling. The total energy is converged within $0.01 \mathrm{meV}$. The calculations are carried out using VASP code. ${ }^{24}$ It is important to point out here that $\mathrm{H}_{2}$ interaction with metal ion complexes arises due to different types of interactions such as electrostatic interactions, orbital interactions, and long range dispersion interactions. As shown recently by Lochan and Head-Gordon in a detailed study, these detailed interaction contributions to the total interaction energy differ for different metal ions. ${ }^{25}$ While first-principles density functional theory (DFT), in principle, should account for all these contributions, in practice, the present exchange correlation functionals are unable to account for long range dispersion interactions completely. In order to find out the accuracy of our density functional theory calculations for describing $\mathrm{H}_{2}$ interaction with graphite, we have compared our results with that of Moller-Plesset (MP2) perturbation based calculations-MP2 theory is known to better describe this interaction including dispersion contribution. Table I shows a comparison of our DFT results with that of MP2 method. ${ }^{26}$ One finds here that while the geometrical parameters between the two schemes are in good agreement with each other, the binding energy of $\mathrm{H}_{2}$ molecule with graphite obtained from DFT method is much less compared to that of MP2 results. This is expected as mentioned earlier due to the absence of long range dispersion interaction in the present functionals, and hence our results will provide a lower bound of $\mathrm{H}_{2}$ interaction on nanopeapod structures. We are not able to use MP2 method for our study of $\mathrm{H}_{2}$ interaction with peapod structure since these calculations are computationally demanding. The remaining results are obtained using density functional method.

First, we compute interaction of $\mathrm{H}_{2}$ molecule with pure carbon nanotube. We have considered the $(10,10)$ nanotube with diameter of about $1.4 \mathrm{~nm}$. This is the smallest nanotube that could accommodate a fullerene $\mathrm{C}_{60}$ molecule as has been observed in experiments. We have optimized the structure of $\mathrm{H}_{2}$ on the nanotube surface and found that the lowest energy structure is $\mathrm{H}_{2}$ bonded to the nanotube surface where molecular axis is parallel to the nanotube axis. The binding energy of $\mathrm{H}_{2}$ on nanotube surface is defined as

$E\left(\mathrm{H}_{2}\right)=\left(\right.$ total energy of nanotube with $\left.\mathrm{H}_{2}\right)$

$$
\text { - (energy of pure nanotube) - (energy of } \left.\mathrm{H}_{2}\right) \text {. }
$$

The binding energy for a parallel orientation is found to be $25 \mathrm{meV}$ as compared to the perpendicular orientation (molecular axis is normal to the nanotube axis) binding energy of $30 \mathrm{meV}$. These values are lower bound for molecular interaction with nanotube, and based on our estimate, the dispersion energy, which is missing in our DFT method, may pro-

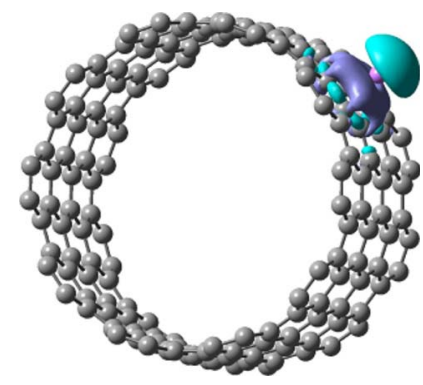

FIG. 1. (Color online) Charge density redistribution plot for $\mathrm{Li}$ coated $(10,10)$ nanotube. $D=D[\mathrm{Li}-$ carbon nanotube $(\mathrm{CNT})$ complex $)-D(\mathrm{CNT})-D(\mathrm{Li})$. Blue and purple represent positive and negative charge distributions correspondingly.

vide additional binding energy of the order of $45 \mathrm{meV}$. This interaction energy is still much smaller compared to the required binding energy of the order of $300 \mathrm{meV}$ for room temperature adsorption and desorption of hydrogen storage process. These results are in good agreement with previous studies and suggest that pure nanotube may not be a good candidate for $\mathrm{H}_{2}$ storage purposes at room temperature and atmospheric pressure. As mentioned earlier, experimental and theoretical results have recently been carried out to investigate whether metal doped carbon structures such as graphenes, fullerenes, and nanotubes could enhance $\mathrm{H}_{2}$ interaction. While early experiments suggested larger wt \% (20 wt \%) for alkali metal coated nanotube, ${ }^{27}$ subsequent results find much smaller capacities of these doped nanotube structures. $^{28,29}$ We have carried out interaction of Li on (10, 10) nanotube surface and found that the lowest energy configuration corresponds to $\mathrm{Li}$ binding on the outside to the center of hexagon ring. The binding energy of Li defined as

$$
\begin{aligned}
E(\mathrm{Li})= & (\text { total energy of Li-nanotube complex }) \\
& -(\text { energy of nanotube })-(\text { energy of } \mathrm{Li})
\end{aligned}
$$

is found to be $1.64 \mathrm{eV}$. This binding strength is different from that binding $\mathrm{Li}$ on graphene $(1.20 \mathrm{eV}$ within GGA functional $^{29}$ ) but is comparable: the small difference arises due to the curvature effect. Indeed, the Li binding energy on $\mathrm{C}_{60}$ surface is found to be about $1.80 \mathrm{eV}$, again demonstrating the effect of curvature on the energetics: the larger the curvature, the higher the binding energy is. The stronger binding of Li compared to molecular hydrogen on the nanotube surface is due to noticeable charge transfer from $\mathrm{Li}$ (about $0.4 e$ ) to the nanotube. Figure 1 shows a redistribution of charge density plot for Li-nanotube complex where one finds excess electron charge density on nanotube surface particularly near the atoms close to Li. Now, we bring the $\mathrm{H}_{2}$ molecule close to the $\mathrm{Li}$ atom on the nanotube surface. We have studied various hydrogen binding sites near Li atom: (a) on top of $\mathrm{Li}$ atom, (b) on top of $\mathrm{C}-\mathrm{C}$ bond along the zigzag direction, (c) on top of $\mathrm{C}-\mathrm{C}$ bond along the armchair direction, (d) on top of $\mathrm{C}$ atom, and (e) in the center of hexagon ring adjacent to Li-hexagon ring (along the tube axis and perpendicular to the tube axis, not shown here). For each binding site, we take two different $\mathrm{H}_{2}$ orientations: tangential and normal to the nanotube surface. We found four different local minimum structures with similar binding energies. The 


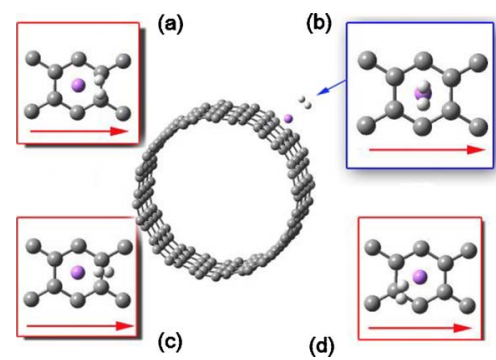

FIG. 2. (Color online) Ball and stick picture of optimized structures of $\mathrm{H}_{2}$ (white balls) on $\mathrm{Li}$ (magenta) coated $(10,10)$ nanotube (gray carbon atoms). Upper right (b) blue structure is an enlarged local top view of center geometry, where hydrogen molecule is on the top of $\mathrm{Li}$ atom with its $\mathrm{H}-\mathrm{H}$ bond parallel to the nanotube surface. Red arrow indicates the tube axis. The other three lowest energy structures are shown in red frame where the hydrogen molecule is binded, (a) with $\mathrm{H}-\mathrm{H}$ bond tangential to the CNT surface and on top of the $\mathrm{C}-\mathrm{C}$ bond along the armchair direction, (c) with $\mathrm{H}-\mathrm{H}$ bond normal to the CNT surface and on top of the C-C bond along the armchair direction, and (d) with $\mathrm{H}-\mathrm{H}$ bond tangential to the CNT surface and on top of one $\mathrm{C}$ atom.

ball and stick structures for these configurations are shown in Fig. 2. One of these configurations is similar to the undoped nanotube surface, as mentioned before. The binding energy of $\mathrm{H}_{2}$ on the Li-nanotube complex is found to be around $180 \mathrm{meV}$-about $150 \mathrm{meV}$ larger compared to that of the undoped nanotube. The increase in binding is due to charge induced dipole interactions originating from charge transfer between $\mathrm{Li}$ and nanotube. It is interesting to point out that in contrast to earlier work by Cabria et al., where they find that $\mathrm{H}_{2}$ binds to the carbon atom on the nanotube surface, our result suggests that lower energy structure also includes where the $\mathrm{H}_{2}$ binds to $\mathrm{Li}$ atom on the nanotube surface. It is equally interesting to point out that $\mathrm{H}_{2}$ binding to the bare $\mathrm{Li}^{+}$ion is much stronger $(0.26 \mathrm{eV})$ (Ref. 30) compared to that on Li-doped nanotube. This could be rationalized due to the fact that $\mathrm{Li}$ on nanotube surface has much reduced localized charge (about 0.42e). Similar conclusions have been observed in metal ion complexes where the charge on $\mathrm{Li}$ atom is reduced substantially compared to its bare charge configuration. ${ }^{25}$ These results suggest that physisorption of $\mathrm{H}_{2}$ on nanotube could be enhanced due to doping in the system, but binding energy is still below what is required for room temperature hydrogen storage purpose. One key understanding that has come out from this and other works is that charge transfer plays an important role on the $\mathrm{H}_{2}$ binding. This suggests that if we could improve further the charge transfer from $\mathrm{Li}$ to nanotube surface, we may be able to further increase the $\mathrm{H}_{2}$ binding. We have recently shown that in fullerene doped carbon nanotubes, known as peapod structures, charge transfer occurs from the nanotube to the fullerene encapsulated inside the nanotube. ${ }^{31}$ In order to study how this could affect our hydrogen binding on the subsequent Li-doped compound, we have carried out extensive calculations for this complex. We have optimized first the peapod structure where $\mathrm{C}_{60}$ molecule is encapsulated inside the $(10,10)$ nanotube. Subsequently, Li is adsorbed on the resulting peapod structure: we have tried $\mathrm{Li}$ on different

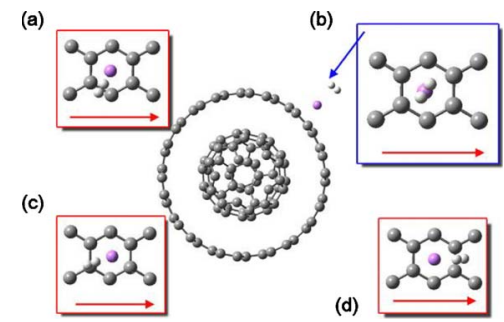

FIG. 3. (Color online) Optimized structure of $\mathrm{H}_{2}$ on Li coated $(10,10)$ nanotube containing $\mathrm{C}_{60}$ molecule inside (nanopeapod structure). Upper right (b) blue structure is an enlarged local top view of center geometry, where hydrogen molecule is on top of $\mathrm{Li}$ atom with its $\mathrm{H}-\mathrm{H}$ bond tangential to the CNT surface. Red arrows indicate tube axis. The other three lowest energy structures of hydrogen binding are shown in red frame, (a) with $\mathrm{H}-\mathrm{H}$ bond normal to CNT surface and on top of $\mathrm{C}-\mathrm{C}$ bond along the zigzag direction, (c) with $\mathrm{H}-\mathrm{H}$ bond normal to CNT surface and on top of $\mathrm{C}$ atom, and (d) with $\mathrm{H}-\mathrm{H}$ bond normal to CNT surface and on top of C-C bond along the armchair direction.

positions on the nanotube surface (outside) such as on top, bridge, and center positions. The lowest energy structure corresponds to where $\mathrm{Li}$ binds to the center of the hexagon on the nanotube surface. The structure is similar to that on nanotube without $\mathrm{C}_{60}$ - however, the binding energy of $\mathrm{Li}$,

$$
\begin{aligned}
E(\mathrm{Li})= & (\text { energy of } \mathrm{Li}-\text { doped peapod structure }) \\
& -(\text { energy of peapod })-(\text { energy of } \mathrm{Li}),
\end{aligned}
$$

is found to be $1.76 \mathrm{eV}$. The Li binding energy on the pure nanotube surface is $1.64 \mathrm{eV}$. The additional $0.12 \mathrm{eV}$ increase in binding of $\mathrm{Li}$ to the nanotube surface is also reflected in the decrease of the distance between $\mathrm{Li}$ and nanotube surface in the nanopeapod structure $(1.71 \AA)$ compared to that in pure nanotube case $(1.76 \AA)$. The noticeable increase in binding of $\mathrm{Li}$ to the nanotube surface results from charge transfer from nanotube to the $\mathrm{C}_{60}$ molecule, which, in turn, facilitates further charge transfer from $\mathrm{Li}$ to nanotube. A charge analysis finds the charge on $\mathrm{Li}$ atom in the peapod structure to be $0.51 e$ compared to $0.42 e$ in the pure nanotube case. It is helpful to recollect here that $\mathrm{C}_{60}$ inside the nanotube acquires about $0.62 e$ from the nanotube. ${ }^{31}$ This makes the nanotube positively charged which helps further charge transfer from $\mathrm{Li}$ to nanotube surface. We then bring $\mathrm{H}_{2}$ molecule toward the peapod complex for the study of the resulting interaction. Again, there are four different binding structures which correspond to a similar lowest energy. The optimized structure of the complex is shown in Fig. 3. Notice that these four configurations are different from those in Fig. 2. The most important result of this Brief Report related to structural and energetics information of $\mathrm{H}_{2}$ interaction with the peapod structure is provided in Table II. The binding energy of $\mathrm{H}_{2}$ molecule within GGA approximation is found to be $217 \mathrm{meV}$ as compared to $\sim 180 \mathrm{meV}$ value of binding energy of $\mathrm{H}_{2}$ on pure Li-nanotube complex. This noticeable increase in $\mathrm{H}_{2}$ binding on the peapod complex is due to increased charge transfer from $\mathrm{Li}$ to the nanotube which is further facilitated by charge transfer from nanotube to the $\mathrm{C}_{60}$ molecule trapped inside the nanotube. We also studied 
the various binding sites of $\mathrm{H}_{2}$ molecule on nanopeapod structure without $\mathrm{Li}$ doped on the outside surface and found the binding energy to be $55 \mathrm{meV}$. Note that this value is also larger compared to similar structures without $\mathrm{C}_{60}$ doped inside $(\sim 30 \mathrm{meV})$. This increase in binding energy is directly due to charge transfer between $\mathrm{C}_{60}$ and nanotube. Although the binding energy of $\mathrm{H}_{2}$ molecule obtained from our calculations are still below the required $300 \mathrm{meV}$ value for room temperature hydrogen purpose, the computed binding is much larger than that of any nanotube based structures studied so far. In addition, our computed value of binding energy represents the lower bound of the "true" binding energy due to lack of dispersion interaction in the present GGA functionals (see Ref. 32 and Table I in this Brief Report). Indeed, the dispersion energy for this system could account additional contribution; in that case, the $\mathrm{H}_{2}$ binding energy with peapod may fall within the required room temperature value for hydrogen storage materials. It is also possible to dope peapod structure with other metals such as $\mathrm{K}$ whose ionization potential is lower than that of $\mathrm{Li}$ where the increase in charge transfer could be larger compared to that in Li which could make $\mathrm{H}_{2}$ interaction stronger. Additionally, it will be of great importance for the study if this increase trend in binding energy of $\mathrm{H}_{2}$ with peapod structures persists for more than one $\mathrm{H}_{2}$ molecule, particularly for achieving the required wt $\%$ for meeting DOE target. Clearly, future theoretical and experimental works are necessary to address these issues.

In summary, we have carried out first-principles density functional calculations based on gradient corrected functional of $\mathrm{H}_{2}$ interaction with pure nanotube, Li-doped nanotube, and Li-doped nanotube peapod structures. Our results show that $\mathrm{H}_{2}$ molecule weakly binds to the nanotube surface without doping, and binding is enhanced with Li-doped nanotube surface. We find four different binding structures which result in a similar binding energy, and the increase in $\mathrm{H}_{2}$ binding is due to the direct interaction between $\mathrm{Li}$ ion and $\mathrm{H}_{2}$ molecule. The molecular binding energy on Li-doped nanotube is much smaller compared to that on the bare $\mathrm{Li}^{+}$ and is in agreement with other metal ion complexes. This is due to the fact that the charge of $\mathrm{Li}$ atom on Li-nanotube
TABLE II. Binding energy of Li and Li-nanotube surface distance on nanotube and nanopeapod structures. Also, the binding energy of $\mathrm{H}_{2}$ molecule and $\mathrm{H}-\mathrm{Li}$ bond distance on nanotube and nanopeapod structures.

\begin{tabular}{lcc}
\hline \hline System studied & Distance $(\AA)$ & Binding energy \\
\hline Li on nanotube & 1.76 & $1.64 \mathrm{eV}$ \\
$\mathrm{Li}$ on nanopeapod & 1.71 & $1.76 \mathrm{eV}$ \\
$\mathrm{H}_{2}$ on Li coated nanotube & 2.10 & $180 \mathrm{meV}$ \\
$\mathrm{H}_{2}$ on Li coated nanopeapod & 2.08 & $217 \mathrm{meV}$ \\
\hline \hline
\end{tabular}

complex is about $0.4 e$ as compared to $+1 e$ charge on bare $\mathrm{Li}$ ion. Encapsulating $\mathrm{C}_{60}$ molecule inside the nanotube provides a different way of increasing charge concentration on $\mathrm{Li}$ adsorbed outside the nanotube surface. We find noticeable charge transfer from nanotube to the $\mathrm{C}_{60}$ molecule which facilitates further charge transfer from $\mathrm{Li}$ to the nanotubethis makes $\mathrm{Li}$ deposited on peapod structure more positive (the Mulliken charge on $\mathrm{Li}$ is found to be about $0.5 e$ ). These results show further increase in $\mathrm{H}_{2}$ binding: the $\mathrm{H}_{2}$ binding energy on the $\mathrm{Li}$ coated peapod structure is found to be over $0.21 \mathrm{eV}$ within GGA approximation. Our computed binding energy provides a lower bound of the true binding energy due to lack of long range dispersion interaction in the present GGA functionals. The increase in $\mathrm{H}_{2}$ binding energy due to $\mathrm{C}_{60}$ encapsulation provides a different way of enhancing $\mathrm{H}_{2}$ binding which may lead to different carbon based materials for hydrogen storage at room temperature (ideal binding energy for this should be around $300 \mathrm{meV}$ ). In addition, doping with other metals such as $\mathrm{K}$ whose ionization potential is lower than that of Li may provide better materials for hydrogen storage. Other issues such as if the enhanced binding energy in peapod structure persists with additional hydrogen molecules (i.e., binding energy per molecule remains on the order of 200-300 meV) and if nanopeapod structures could provide required gravimetric wt $\%$ will be interesting to explore in future studies.

This work is supported by National Science Foundation (NSF) NIRT under Award No. 0403789 (N.K. and S.K.N).

\footnotetext{
${ }^{1}$ A. C. Dillon et al., Nature (London) 386, 377 (1997).

${ }^{2}$ R. Coontz and B. Hanson, Science 305, 957 (2004).

${ }^{3}$ S. P. Chan et al., Phys. Rev. Lett. 87, 205502 (2001).

${ }^{4}$ K. Tada et al., Phys. Rev. B 63, 155405 (2001).

${ }^{5}$ Y. Miura et al., J. Appl. Phys. 93, 3395 (2003).

${ }^{6}$ G. Lu et al., Phys. Rev. B 68, 205416 (2003).

${ }^{7}$ P. Dubot and P. Cenedese, Phys. Rev. B 63, 241402(R) (2001).

${ }^{8}$ E. C. Lee et al., Phys. Rev. B 66, 073415 (2002).

${ }^{9}$ O. Gulseren et al., Phys. Rev. B 66, 121401(R) (2002).

${ }^{10} \mathrm{http} / / / \mathrm{www} 1 . e e r e . e n e r g y . g o v / h y d r o g e n a n d f u e l c e l l s / s t o r a g e / t e c h$ status.html.

${ }^{11}$ A. Zuttel, Mater. Today 6, 24 (2003).

${ }^{12}$ S. Orimo et al., J. Alloys Compd. 356, 716 (2003).

${ }^{13}$ G. G. Tibbetts et al., Carbon 39, 2291 (2001).

${ }^{14}$ C. M. Brown et al., Chem. Phys. Lett. 329, 311 (2000).

${ }^{15}$ B. K. Pradhan et al., Physica B 323, 115 (2002).

${ }^{16}$ D. G. Narehood et al., Phys. Rev. B 67, 205409 (2003).

${ }^{17}$ T. Yildirim and S. Ciraci, Phys. Rev. Lett. 94, 175501 (2005).
}

${ }^{18}$ Q. Sun et al., J. Am. Chem. Soc. 127, 14582 (2005).

${ }^{19}$ Q. Sun et al., J. Am. Chem. Soc. 128, 9741 (2006).

${ }^{20}$ I. Cabria et al., J. Chem. Phys. 123, 204721 (2005).

${ }^{21}$ J. P. Perdew et al., Phys. Rev. B 46, 6671 (1992).

${ }^{22}$ D. Vanderbilt, Phys. Rev. B 41, 7892 (1990).

${ }^{23}$ P. E. Blochl, Phys. Rev. B 50, 17953 (1994).

${ }^{24}$ G. Kresse and J. Hafner, Phys. Rev. B 47, 558 (1993).

${ }^{25}$ R. C. Lochan and M. Head-Gordon, Phys. Chem. Chem. Phys. 8, 1357 (2006).

${ }^{26}$ Y. Okamoto and Y. Miyamoto, J. Phys. Chem. B 105, 3470 (2001).

${ }^{27}$ P. Chen et al., Science 285, 91 (1999).

${ }^{28}$ M. Khantha et al., Phys. Rev. B 70, 125422 (2004).

${ }^{29}$ Z. H. Zhu et al., Carbon 42, 2509 (2004).

${ }^{30}$ B. K. Rao and P. Jena, Europhys. Lett. 20, 307 (1992).

${ }^{31}$ R. Pati et al., J. Appl. Phys. 95, 694 (2004).

${ }^{32}$ X. Wu et al., J. Chem. Phys. 115, 8748 (2001). 